Review article

\title{
Lung transplantation from donation after controlled cardiocirculatory death. Systematic review and meta-analysis
}

\author{
Alessandro Palleschi a , Lorenzo Rosso a,b ${ }^{\mathrm{a}}$, Valeria Musso ${ }^{\mathrm{c}, *}$, Arianna Rimessi ${ }^{\mathrm{c}}$, \\ Gianluca Bonitta ${ }^{\mathrm{d}}$, Mario Nosotti ${ }^{\mathrm{a}, \mathrm{d}}$ \\ a Thoracic Surgery and Lung Transplantation Unit, Fondazione IRCCS Ca' Granda Ospedale Maggiore Policlinico of Milan, Milan, Italy \\ b Dipartimento di Scienze della Salute, Università degli studi di Milano, Milan, Italy \\ c Università degli studi di Milano, Milan, Italy \\ d Dipartimento di Fisiopatologia medico-chirurgica e dei Trapianti, Università degli studi di Milano, Milan, Italy
}

\section{A R T I C L E I N F O}

\begin{abstract}
A B S T R A C T
Background: The interest in donation after cardiocirculatory death (DCD) donors for lung transplantation (LT) has been recently rekindled due to lung allograft shortage. Clinical outcomes following DCD have proved satisfactory. The aim of this systematic review is to provide a thorough analysis of published experience regarding outcomes of LT after controlled DCD compared with donation after brain death (DBD) donors.

Methods: We performed a literature search in Cochrane Database of Systematic Reviews, PubMed and Web of Science using the items "lung transplantation" AND "donation after circulatory death" on November 1,2018. The full text of relevant articles was evaluated by two authors independently. Quality assessment was performed using the NIH protocol for case-control and case series studies. A pooled Odds ratio (OR) and mean differences with inverse variance weighting using DerSimonian-Laird random effect models were computed to account for between-trial variance $(\tau 2)$.

Results: Of the 508 articles identified with our search, 9 regarding controlled donation after cardiac death (cDCD) were included in the systematic review, including 2973 patients (403 who received graft from DCD and 2570 who had DBD). Both 1-year survival and 2 and 3-grade primary graft dysfunction (PGD) were balanced between the two cohorts ( $\mathrm{OR}=1.00$ and 1.03 respectively); OR for airway complications was 2.07 against $\mathrm{CDCD}$. We also report an $\mathrm{OR}=0.57$ for chronic lung allograft dysfunction (CLAD) and an $\mathrm{OR}=0.57$ for 5-year survival against CDCD.

Conclusions: Our meta-analysis shows no significant difference between recipients after CDCD or DBD regarding 1-year survival, PGD and 1-year freedom from CLAD. Airway complications and long-term survival were both related with transplantation after $\mathrm{CDCD}$, but these statistical associations need further research.
\end{abstract}

(c) 2019 Elsevier Inc. All rights reserved.

\section{Contents}

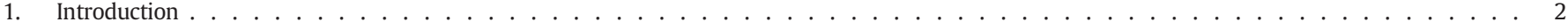

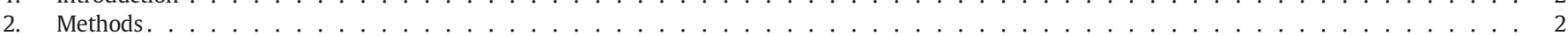

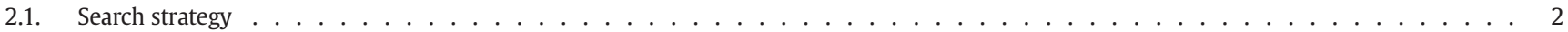

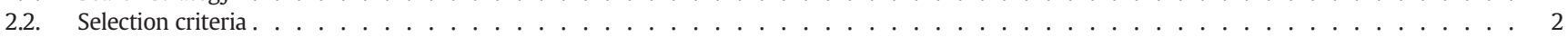

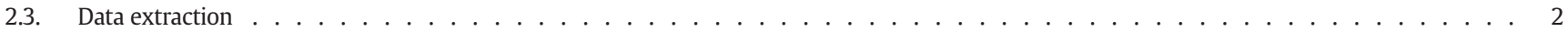

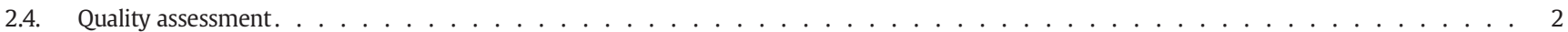

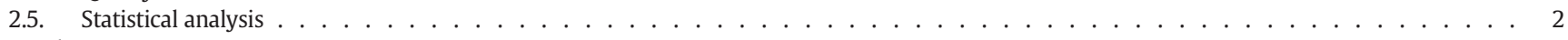

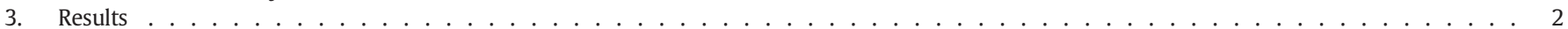

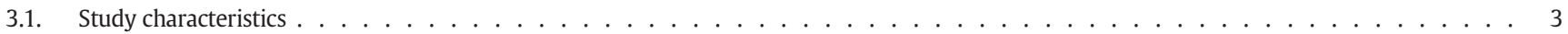

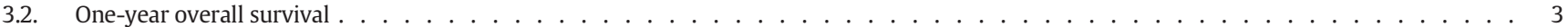

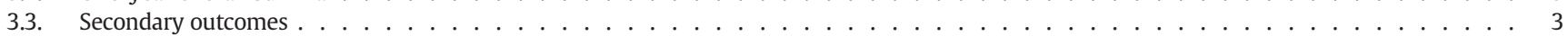

\footnotetext{
* Corresponding author at: Fondazione IRCCS Ca'Granda Ospedale Maggiore Policlinico, Via Francesco Sforza, 3520122 Milan, Italy.

E-mail address: valeria.musso@unimi.it (V. Musso).
} 


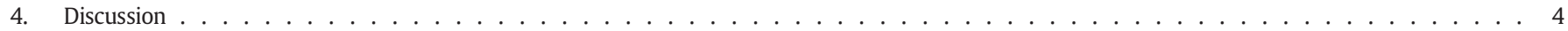

Appendix A. Supplementary data. . . . . . . . . . . . . . . . . . . . . . . . . . . . . . . . .

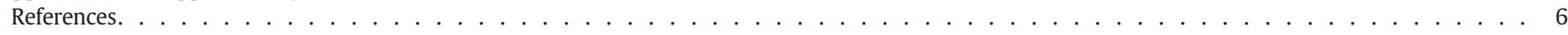

\section{Introduction}

The first attempt of human lung transplantation (LT), as well as the first LT with long-term survival, was carried out with donation after cardiocirculatory death (DCD) donors [1,2]. Notwithstanding, transplantations from DCD donors were nearly abandoned in favor of transplantations from brain death donors in the early seventies. Twenty years later, the interest in DCD donors has been rekindled for two main reasons: the persistent lung allograft shortage and the hypothesis according to which lungs from DCD are free from the hemodynamic perturbations caused by brain death. This last assumption has not been clearly demonstrated yet. Nevertheless, several preclinical and clinical studies stated that lung parenchyma survives fairly well after circulatory arrest [3-6]. In the "modern era", clinical outcomes following DCD LT have proved acceptable; several centers introduced original protocols to implement such type of donation. The International Society for Heart and Lung Transplantation (ISHLT) instituted the DCD Registry in 2011; ten centres from Australia, Europe and North America were invited to participate. The first published report from the DCD Registry concluded that LT using controlled DCD had excellent outcome [7]. The 6th International Conference on Organ Donation, which was held in Paris in 2014, discussed the reports from one uncontrolled and eleven controlled DCD programs: a list of recommendations was provided [8]. Such recommendations meant to harmonize terminology and basic concepts that appeared to be fairly inhomogeneous among the considered twelve DCD programs.

The aim of the present systematic review is to collect published experience to analyze the outcome of LT from DCD compared with donation after brain death (DBD) donors.

\section{Methods}

\subsection{Search strategy}

A literature search in Cochrane Database of Systematic Reviews, PubMed, and Web of Science was performed by a scientific librarian using the items: lung transplantation AND donation after circulatory death. The search queries are reported in the supplemental material. Searches were not limited by date or publication status; English language was requested. The date of search was November 1, 2018. To identify additional studies we hand-searched the reference lists of eligible papers.

\subsection{Selection criteria}

For this review we considered all the original studies, ranging from case series to randomized trials. The selected papers had to focus on lung transplantation (including lobe, single-lung, and bilateral transplants); heart-lung transplantation was excluded. We planned to include studies of comparisons between transplantation from DCD versus DBD donors.

Titles and abstracts of papers identified through searching were screened; the full text of relevant articles was obtained and screened. Two authors (AR, AP) evaluated each article independently; discrepancies were settled by consensus involving a third author (MN). In case of overlapping reports from the same center the most recent paper was considered; data from multi-institutional studies were carefully checked to avoid duplicates. This study is registered in the PROSPERO
International Prospective Register of Systematic Reviews, registration number CRD42018112152.

The primary end-point was 1-year overall survival; secondary endpoints were primary graft dysfunction (PGD), intensive care unit stay, length of hospital stay, 30-day mortality, airway complications, 1-year overall survival free from chronic lung allograft dysfunction (CLAD) and 5-year overall survival.

\subsection{Data extraction}

The following data were extracted: names of the first author, year of publication, sample size, details of patients' characteristics, PGD rate, length of stay in intensive care unit and in hospital, 30-day mortality, airway complication rate, 1 -year free from CLAD rate, survival at 1 and 5 years. The corresponding authors of the selected studies were contacted to obtain unpublished and/or updated data. Data from eligible articles as well as the update were collected in a dedicated database.

\subsection{Quality assessment}

The included studies were assessed for quality using the protocol for case-control and case series studies implemented by the National Institute of Health - National Heart, Lung, and Blood Institute (https:// www.nhlbi.nih.gov/health-pro/guidelines/in-develop/cardiovascularrisk-reduction/tools/cohort). The tools were independently used by the two reviewers (AP, AR) $(\mathrm{k}=0.95)$. Studies that scored "No" for no more than one item were assessed as being of good quality; those that scored "No" for 2 or 3 items were assessed as being of fair quality.

\subsection{Statistical analysis}

The results of the systematic review were summarized qualitatively into frequentist meta-analysis. We pooled Odds ratios (ORs) and mean differences with inverse variance weighting using DerSimonian-Laird random effect models to account for between-trial variance ( $\tau 2$ ) [9]. Heterogeneity among the trials was quantified by $\mathrm{I}^{2}$-index and Cochran's $Q$ test [10], considering significant heterogeneity when $\mathrm{p}$ value was $<0.10$ or $\mathrm{I}^{2}$-index was $>50 \%$ [11]). A leave-one-out sensitivity analysis was performed by iteratively removing one study at a time to confirm that our findings were not driven by any single study. The 95\% confidence intervals (CIs) were based on normal approximation and $Z$-score test was performed for assessment of the overall treatment effect. The 95\% confidence intervals for $\mathrm{I}^{2}$ index were calculated according to Higgins and Thompson [12]. The 95\% prediction intervals for the treatment effect of a new study are calculated according to Borenstein [10]. Two-sided p-values were considered statistically significant when $\mathrm{p}<0.05$. In accordance with Cochrane guidance, we did not investigate publication bias because our search considered less than ten studies for each data comparison. Standard deviations for continuous outcome variables were calculated according to Hozo, if necessary [13]. All analyses and graphs were carried out using R software (version 3.2.2) [14].

\section{Results}

The literature search yielded a total of 508 papers, 429 of which were excluded after title matching or abstracts review. Nine of the remaining 79 studies met the inclusion criteria and were included in the systematic review. Those 9 papers were eventually considered for the meta- 
analysis including 2973 patients [15-23]. Fig. 1 shows the flow diagram detailing paper screening and selection. The main characteristics of the selected study are presented in Table 1.

\subsection{Study characteristics}

All selected papers reported single center experience except Levvey who collected the experiences of the Australian centers [18]. The majority of the studies were judged "fair" after quality assessment (Fig. 1 in supplementary materials). In summary, the selected studies included 403 patients who received graft from DCD and 2570 who had DBD. All the selected analytic retrospective studies were restricted to controlled DCD according to the Maastricht classification; Table 2 shows some specific points that characterized the retrieval protocols adopted from the transplantation centres.

\subsection{One-year overall survival}

The estimated pooled odds ratio of 1-year overall survival cumulative incidence resulting from 8 studies with a total of 2620 patients was 1.00 (95\% CI $=0.70-1.44 ; \mathrm{p}=0.973$ ). Prediction lower and upper limits were 0.43 and 2.30 , respectively. The heterogeneity was low and not significant $\left(\mathrm{I}^{2}=19.2 \%, 95 \% \mathrm{CI}=0.0-61.8 .7 \% ; \mathrm{p}=0.278\right)$ with $\tau^{2}=0.07$ (Fig. 2). The sensitivity analysis supported the robustness of these results (Fig. 2 in the supplementary materials).

\subsection{Secondary outcomes}

The selected studies reported PGD with various modalities; focusing on highest grades (PGD grade $2-3$ in the first $72 \mathrm{~h}$ ), the estimated pooled odds ratio resulting from 7 studies with a total of 1787 patients was 1.03 (95\% CI =0.74-1.44; $\mathrm{p}=0.867$ ). Prediction lower and upper limits were 0.66 and 1.60 , respectively. The heterogeneity was low and not significant, $\mathrm{I}^{2}=0.0 \%$ (Fig. 3 in the supplementary material). The sensitivity analysis confirmed the robustness of these results (Fig. 3 in the supplementary material).

Six studies reported the length of intensive care unit (ICU) stay that ranged from 3 to 16.3 days in DCD cohorts versus 3 to 14.4 days in DBD cohorts. There was no difference in terms of length of stay in ICU; the heterogeneity among studies was high (Fig. 7, 8 in the supplementary material). The mean length of hospital stay was reported in 5 studies; DCD cohorts ranged from 17 to 41 days, BDB cohorts ranged from 20 to 38.1 days. There was no difference in terms of length of hospital stay; the heterogeneity among studies was high (Fig. 9, 10 in the supplementary material).
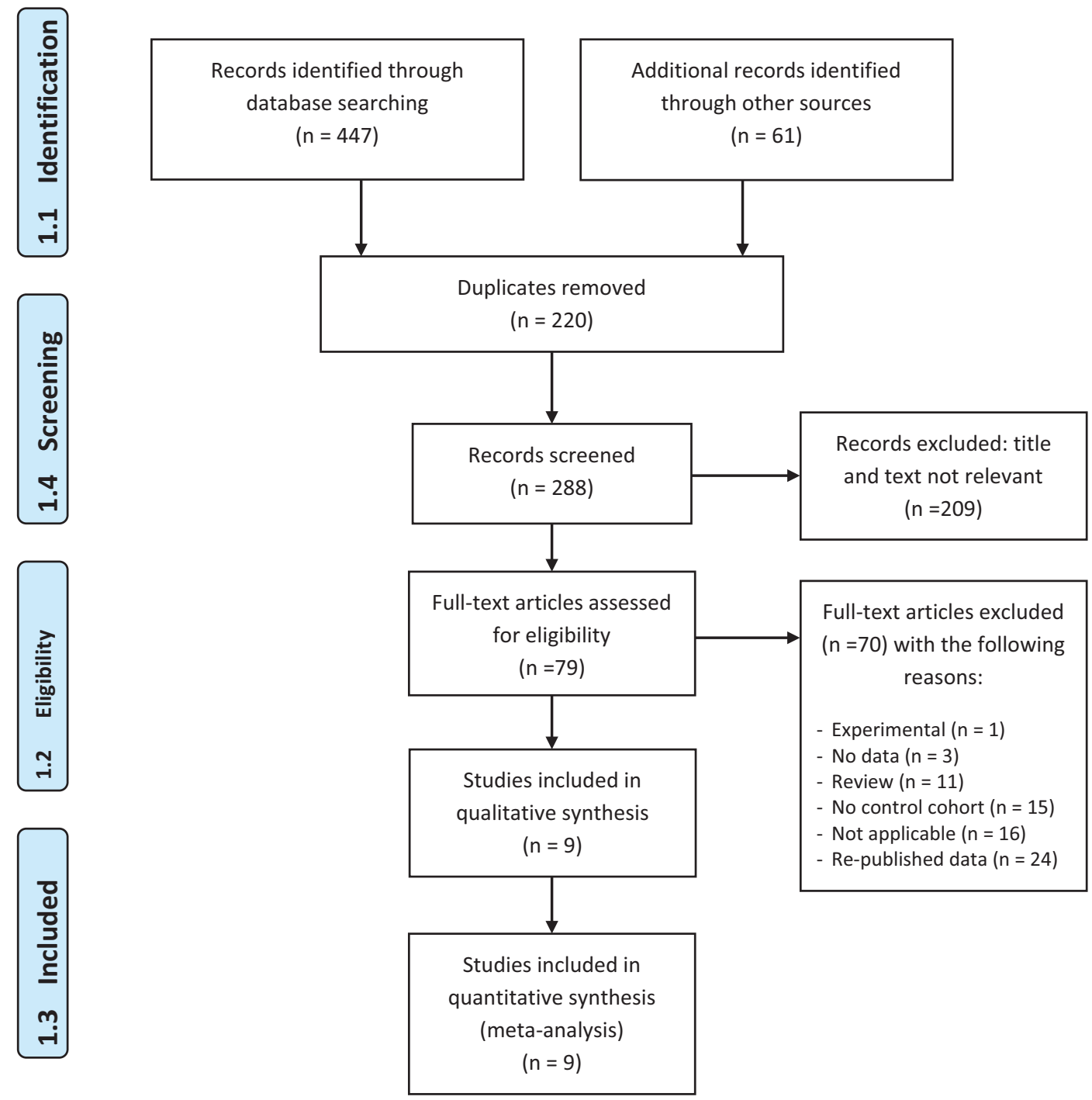

Fig.1. PRISMA Flow Diagram. 
Table 1

Studies selected for review.

\begin{tabular}{|c|c|c|c|c|c|c|c|c|c|c|c|}
\hline $\begin{array}{l}\text { Author and } \\
\text { year }\end{array}$ & Location & $\begin{array}{l}\text { Time } \\
\text { frame }\end{array}$ & Study design & DCD & DBD & $\begin{array}{l}\text { Recipient } \\
\text { sex }(\% \mathrm{M}) \\
(\mathrm{DCD} / \mathrm{DBD})\end{array}$ & $\begin{array}{l}\text { Recipient age } \\
\text { (mean) } \\
(\mathrm{DCD} / \mathrm{DBD})\end{array}$ & $\begin{array}{l}\text { BMI } \\
(\mathrm{DCD} / \mathrm{DBD})\end{array}$ & $\begin{array}{l}\text { Bilateral } \\
\text { LT (\%) } \\
(\mathrm{DCD} / \mathrm{DBD})\end{array}$ & Q. A. & Update \\
\hline Puri, [15] & St. Louis, MO & 2003-2008 & $\begin{array}{l}\text { Analytic retrospective } \\
\text { cohort, single center }\end{array}$ & 11 & 282 & N.R. & $54 / 48$ & $21.6 / 22.6$ & $100 / 95.4$ & Fair & No \\
\hline De oliveira, [16] & Madison, WI & 1993-2009 & $\begin{array}{l}\text { Analytic retrospective } \\
\text { cohort, single center }\end{array}$ & 18 & 406 & $61 / 63$ & $47 / 50$ & $23 / 24$ & $50.0 / 36.0$ & Fair & No \\
\hline Van de wauwer, [17] & Groningen, Nl & 2005-2009 & $\begin{array}{l}\text { Analytic retrospective } \\
\text { cohort, single center }\end{array}$ & 35 & 77 & $34 / 51$ & $54 / 53$ & N.R. & $85.7 / 71.4$ & Fair & No \\
\hline Levvey, [18] & Australia & 2006-2011 & $\begin{array}{l}\text { Analytic retrospective } \\
\text { cohort, multi-center }\end{array}$ & 133 & 905 & $56 / 50$ & $43 / 46$ & N.R. & $95.8 / 86.4$ & Good & Yes \\
\hline Sabashnikov, [19] & Harefield, UK & 2007-2013 & $\begin{array}{l}\text { Analytic retrospective } \\
\text { cohort, propensity score } \\
\text { matching, single center }\end{array}$ & 60 & 120 & $55 / 43$ & $43 / 41$ & $21.1 / 22.3$ & $97.9 / 99.0$ & Good & No \\
\hline Ruttens, [20] & Leuven, Be & 2007-2013 & $\begin{array}{l}\text { Analytic retrospective } \\
\text { cohort, single center }\end{array}$ & 59 & 331 & $62 / 48$ & $51 / 50$ & N.R. & $98 / 92$ & Fair & No \\
\hline Costa, [21] & New York, NY & 2007-2016 & $\begin{array}{l}\text { Analytic retrospective } \\
\text { cohort, single center }\end{array}$ & 46 & 237 & $43 / 58$ & $58 / 57$ & N.R. & $50 / 47$ & Fair & Yes \\
\hline Inci, [22] & Zurich, Ch & 2012-2017 & $\begin{array}{l}\text { Analytic retrospective } \\
\text { cohort, single center }\end{array}$ & 21 & 151 & $57 / 55$ & $49 / 50$ & $21 / 21$ & $100 / 97$ & Fair & No \\
\hline Barbero, [23] & Cambridge, UK & 2009-2015 & $\begin{array}{l}\text { Analytic retrospective } \\
\text { cohort, single center }\end{array}$ & 23 & 163 & $51 / 59$ & $58 / 50$ & $24 / 24$ & $70 / 82$ & Fair & No \\
\hline
\end{tabular}

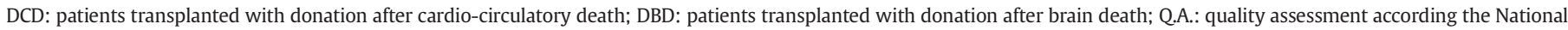
Institute of Health. N.R.: not reported.

Six papers reported the frequency of the airway complications: the prevalence ranged from 4.7 to 27.7 in DCD cohorts versus 1.3 to 18.8 in DBD cohorts. The estimated pooled odds ratio of airway complications resulting from 4 studies with a total of 962 patients was 2.07 (95\% CI $=1.09-3.94 ; \mathrm{p}=0.026)$. Prediction lower and upper limits were 0.51 and 8.47 , respectively. The heterogeneity was $\mathrm{I}^{2}=0.0 \%$ (Fig. 4 in the supplementary material). The sensitivity analysis shows that by omitting the De Olivera's study the overall effect test becomes no longer significant ( 0.147 vs 0.026 ) (Fig. 4 in the supplementary material).

Data on 1-year survival rate free from CLAD were available in 6 studies; the estimated pooled odds ratio resulting from 5 studies with a total of 1002 patients was 0.57 ( $95 \% \mathrm{CI}=0.19-1.72 ; \mathrm{p}=0.321)$. Prediction lower and upper limits were 0.01 and 23.63 , respectively. The heterogeneity was moderate but not significant, $\mathrm{I}^{2}=34.1 \%$ (Fig. 5 in the supplementary material). The sensitivity analysis showed that by omitting Van De Wauwer's study the overall effect test becomes statistically significant ( 0.321 vs 0.041 ) and odds ratio decreases from 0.57 to 0.38 with upper limit of $95 \%$ confidence interval equal to 0.96 (Fig. 5 in the supplementary material).

Five papers reported the 5-year survival rate of 1631 patients; the estimated pooled odds ratio was $0.57(95 \% \mathrm{CI}=0.43-0.76$; $\mathrm{p}<0.001)$.
The prediction lower and upper limits were 0.36 and 0.91 , respectively. The heterogeneity was low, $\mathrm{I}^{2}=0.0 \%$ (Fig. 2). The sensitivity analysis documented the robustness of the results (Fig. 6 in the supplementary material).

\section{Discussion}

The number of papers published on DCD has been increasing as a result of a growing interest in this peculiar type of donation. Bearing in mind that randomized controlled trials are almost impossible to carry out in this context, some reviews were published with the aim of getting some scientific evidence on lung transplantation. In 2008, Oto published a review that collected 8 papers ( 39 patients); the author concluded that LT after controlled DCD had adequate early clinical outcomes, whereas the use of uncontrolled DCD seemed to be related to high incidence of PGD [24]. Wigfield published in 2014 a narrative review confirming the good early outcome of LT after DCD; this paper underlined the differences among protocols, namely in the use of ex-vivo lung perfusion (EVLP) [25]. A systematic review and metaanalysis was published by Krutsinger and collaborators in 2015; this well conducted study collected 11 articles, 6 of which entered the meta-analysis (DCD cohort: 271 recipients; DBD cohort: 2369) [26].

Table 2

Main characteristics of the controlled DCD protocols.

\begin{tabular}{|c|c|c|c|c|c|c|}
\hline Author and year & "Hands off" time (min) & Interval 2 (min) & Interval 4 (min) & Interval 3 (min) & Lung reconditioning & Ischemic time (min) \\
\hline Puri, [15] & 5 & n.a. & n.a. & n.a. & no & $266^{\mathrm{a}}$ \\
\hline De Oliveira, [16] & 5 & n.a. & n.a. & 30 & no & $392^{\mathrm{b}}$ \\
\hline Van De Wauwer, [17] & 5 & 17 & n.a. & 29 & no & $458^{c}$ \\
\hline Levvey, [18] & $2-5$ & 18 & 21 & 35 & no & $254^{\mathrm{d}}$ \\
\hline Sabashnikov, [19] & 5 & n.a. & n.a. & n.a. & occasional (EVLP or OCS) & $346^{\mathrm{a}}$ \\
\hline Ruttens, [20] & 5 & n.a. & n.a. & 21 & no & $314^{\mathrm{a}}$ \\
\hline Costa, [21] & 5 & 23 & 14 & 32 & no & n.a. \\
\hline Inci, [22] & 10 & 17 & 37 & 42 & Occasional (EVLP) & n.a. \\
\hline Barbero, [23] & 5 & 14 & 34 & n.a. & no & n.a. \\
\hline
\end{tabular}

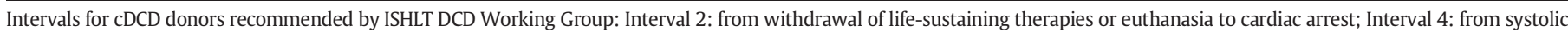

blood pressure $<50 \mathrm{mmHg}$ to cold flush; Interval 3: from withdrawal of life-sustaining therapies or euthanasia to cold flush.

a Absence of precise indication.

b "Cold ischemic time".

c From cold flush to the reperfusion.

d From cardiac arrest to first lung reperfusion. 


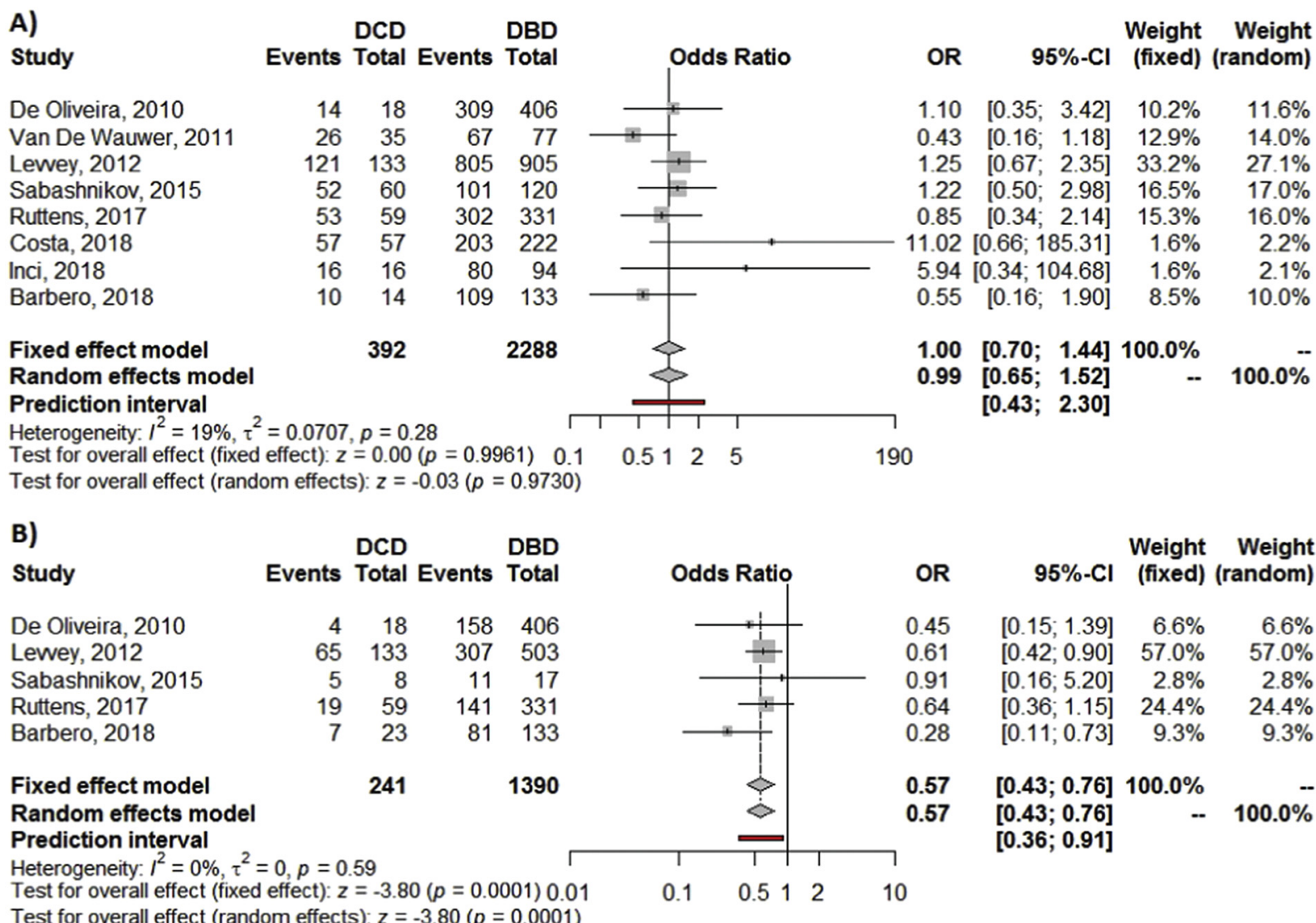

Fig.2. A) Forest plot of pooled analysis for 1-year survival after lung transplantation. B) Forest plot of pooled analysis for 5-year survival.

The authors found that there was no difference in 1-year overall survival rate between patients who received graft from DCD or DBD; PGD and acute cellular rejection rates were also similar between the two patients' cohorts [26]. The Erasmus review formulated a number of recommendations analyzing 12 DCD programs [8]. The first 7 general recommendations have, for the most part, a low degree of evidence; all remaining 6 recommendations addressing the uncontrolled DCD were given evidence grade " $D$ ". In this context it is evident that great efforts will be necessary to bring DCD in the area of evidence based medicine.

The present review gathered nine analytic retrospective studies, almost doubling the DCD cohort analyzed by Krustinger. Originally, we were not going to restrict our research on the basis of DCD Maastricht category, but the studies with control arms only included category III donors; therefore, this review is focused on controlled DCDs. The recipient selections were inhomogeneous: Puri consciously attempted to use the DCD graft for recipients considered to be at baseline risk for perioperative complications [15]; on the contrary, De Oliveira's recipient cohort nearly always included the sickest listed patients [16]. This apparently dichotomous behavior probably reflects the overall impression that lungs from DCD are somehow inferior in quality to those obtained from DBD. Recent studies openly stated that recipient selection according to donor type was avoided; or, at least, the lack of selection can be probably inferred from the analysis of the characteristics of the cohorts [17-23]. Donor selection criteria were not clearly stated in some papers $[17,20,21,23]$, whereas others accepted donors with "extended criteria" such as smoking history of $>20$ pack/years, intensive care unit stay $>5$ days, $\mathrm{PaO} 2 / \mathrm{FiO} 2<400 \mathrm{mmHg}$, and abnormal chest X-ray $[18,19,22]$. Puri did not accept DCD donors defined as "marginal" [15]. It is obvious that this inhomogeneity in recipient as well as donor selection, together with the typical distortions of observational studies, created some confusion and enrolment bias were possible.
The "hands off" time was 5 minutes for almost all centers except for Zurich (10 minutes) and some Australian centers (2 minutes) [18,22]. "Hands off" times of this entity are not considered capable of significantly impacting the results, while precise limits of the agonic time were established through different protocols. The Groningen protocol [17] limited the agonic time to 60 minutes, while Australian protocols ranged from 60 to 90 minutes [18]; the two British protocols were more liberal, extending the acceptability up to 2 hours following withdrawal of life support or up to 1 hour following onset of warm ischemic period $[19,23]$. When reported, the mean duration of interval 2 (from withdrawal of life-sustaining therapies to cardiac arrest) ranged from 14 to 23 minutes; these time intervals were safely below the stated limits (Table 2). How extensive the warm ischemic time may be to prove acceptable is yet to be determined. We had a favorable 1-year outcome in recipients of lungs procured from DCD donors with extended warm ischemic time: average 157 minutes (range 118-179) (personal communication at the $8^{\text {th }}$ International Meeting on Transplantation from DCD, Milan on September 13-14, 2018).

The majority of the procurement protocols included rapid sternotomy and pulmonary arterial cannulation for the delivery of the preservation solution [16-20,22]; some authors preferred topical cooling immediately after sternotomy $[15,21,23]$. Both procedures present advantages and drawbacks; topical cooling guarantees a rapid downturn of cellular metabolism (even though the lung parenchyma has the property to decouple ischemia from hypoxia and all protocols provide for the maintenance of the ventilation during the procurement); on the other hand, avoiding the flooding of the pleural space allows an accurate inspection of the parenchyma.

Most of the centres did not evaluate the lungs with EVLP after procurement. This fact is easily explained, as a proper functional evaluation was achievable shortly before the withdrawal of life support. Nevertheless, EVLP is an appropriate tool for the evaluation of graft function 
when extended criteria donors are considered. Moreover, the use of EVLP is advised in case of interval between withdrawal of life support therapy and cardiac arrest greater than 60 minutes or if pulmonary edema, poor compliance or high-risk history are likely present [27].

The odds ratio for 1-year overall survival (primary end-point of current meta-analysis) was perfectly balanced between the two cohorts, even though a "conservative" random model was used. These findings strengthen the result obtained by Krustinger and collaborators [26], considering that 3 new analytic retrospective cohort studies were published along with 2 adjourned papers. Our result was also reinforced with the leave-one-out sensitivity analysis that evaluated the ambiguity in the output distributed to each selected study considered as source of uncertainty [28]. We think that it is now possible to state with reasonable certainty that controlled DCD guarantees 1 -year overall survival after LT not less than DBD.

Our pooled analysis shows that there is no difference in grade 2-3 PGD rates between recipients of DCD and DBD. Despite this, the result was straightened by heterogeneity and sensitivity tests; it is important to underline that PGD data were reported in different ways and, as a consequence, this result must be taken with caution. Our result, as well as Krustinger's, supported the hypothesis that Maastricht class III donors offer lung grafts without the negative impact caused by brain death. Such hypothesis has been well demonstrated in animal studies where catecholamine discharge, inflammatory response and higher immunogenicity caused by brain death could result in acute lung injury. The ischemia reperfusion injury affects DBD as well as DCD organs but the warm ischemic time after the cardiac arrest can worsen inflammation and vascular leakage. The early resumption of ventilation, the preconditioning effect and the possibility of using EVLP seem to mitigate the noxae for the lungs during the DCD process. Despite the need to accumulate further (possibly prospective) clinical studies, it is now becoming increasingly evident that controlled DCD guarantees a short-term outcome which overlaps at least with that obtained from LT with DBD.

One-year freedom from CLAD was never tested in a meta-analysis; our results documented balanced odds ratio for this parameter. The result must be taken with caution considering that cohort size and heterogeneity tests are on the edge of acceptability. On the other hand, it is well known that "autonomic storm" takes place during brain death; after initial increase in the parasympathetic tone, an early massive sympathetic outflow is produced. Organs, which are exposed to intense sympathetic stimulation, face the release of adhesion molecules, cytokines, chemokines as well as overexpression of histocompatibility antigens [29]. Theoretically, the absence of this over-expression could help to reduce the rate of CLAD and improve medium-term survival in LT from controlled DCD.

A debated point in DCD is the possibility of airway complications as a consequence of main bronchi hypoperfusion during low-flow state. Our pooled analysis fuels this concern, stating that the odds ratio for airway complications was 2 against LT with DCD. This result deserves few considerations: there is institutional variability in the definition of airway complications and the cohorts were of relatively small size, despite heterogeneity being the lowest possible. In addition, such negative result for DCD disappeared if one study was omitted in the sensitivity analysis [16]. Considering how experimental studies pointed out the impaired healing of the bronchial anastomosis in LT from DCD, this item will require special attention in future reports; increasing the effort for homogeneous definitions and careful analysis of confounding factors will also prove necessary [30].

Lastly, long-term survival tested with pooled analysis was significantly in favor of LT with DBD. The interpretation of this result is problematic; 5-year survival could be lower in controlled DCD recipients as a consequence of bias in the lungs allocation: some authors preferred DCD graft in patients with severe clinical condition (patients judged too compromised to wait for DBD graft); other authors selected patients with low priority who have to wait a long time for a
DBD graft. It is possible that long-term survival may be more affected by the pre-existing conditions of the recipient and his/her management than by the type of donor [31]. In any case, the heterogeneity of the 5 included studies is extremely low; therefore, a consistent number of new trials is needed to alter the disadvantage of the DCD recipients.

Limitations of this study include the retrospective nature of the selected papers. The cohorts have been matched with propensity score only in one article; the number of patients was small, with only 392 recipients in controlled DCD cohort. Allocation bias between the two cohorts was highly probable, namely with regard to recipient selection. Pooled analysis was inconclusive for ICU and hospital stay because of extreme heterogeneity. Lastly, 1-year follow-up is a relatively short timeframe when referred to CLAD; in addition, old papers reported bronchiolitis obliterans syndrome rather than CLAD.

In conclusion, this meta-analysis indicates that there is no significant difference in 1-year overall survival, PGD and 1-year freedom from CLAD between recipients of controlled DCD or DBD lungs. Conversely, a warning is mandatory for airway complications and 5-year survival, despite the effective responsibility of the donor type being questionable. In this context, it remains vital for centers to continue publishing their experience on DCD to achieve high quality evidences.

\section{Declaration of Competing Interest}

None.

The authors have no conflict of interest to declare

\section{Appendix A. Supplementary data}

Supplementary data to this article can be found online at https://doi. org/10.1016/j.trre.2019.100513.

\section{References}

[1] Hardy JD, Webb WR, Dalton ML, Walker GR. Lung Homotransplantation in man: report of the initial case. JAMA J Am Med Assoc 1963;186:1065-7465. https://doi.org/ 10.1001/jama.1963.63710120001010.

[2] Group TLT. Unilateral lung transplantation for pulmonary fibrosis. Toronto Lung Transplant Group. N Engl J Med 1986;314:1140-5140. https://doi.org/10.1056/ NEJM198605013141802.

[3] Egan TM, Lambert CJ, Reddick R, Ulicny KS, Keagy BA, Wilcox BR. A strategy to increase the donor pool: use of cadaver lungs for transplantation. Ann Thorac Surg 1991:52:1113-2013. https://doi.org/10.1016/0003-4975(91)91290-C.

[4] Van Raemdonck DEM, Jannis NCP, Rega FRL, De Leyn PRJ, Flameng WJ, Lerut TE. Extended preservation of ischemic pulmonary graft by postmortem alveolar expansion. Ann Thorac Surg 1997;64:801-8. https://doi.org/10.1016/S0003-4975(97)00627-9.

[5] Inci I, Zhai W, Arni S, Inci D, Hillinger S, Lardinois D, et al. Fibrinolytic treatment improves the quality of lungs retrieved from non-heart-beating donors. J Heart Lung Transplant 2007;26:1054-6054. https://doi.org/10.1016/j.healun.2007.07.033.

[6] Valenza F, Citerio G, Palleschi A, Vargiolu A, Fakhr BS, Confalonieri A, et al. Successful transplantation of lungs from an uncontrolled donor after circulatory death preserved in situ by alveolar recruitment Maneuvers and assessed by ex vivo lung perfusion. Am J Transplant 2016;16:1312-8312. https://doi.org/10.1111/ajt.13612.

[7] Cypel M, Levvey B, Van Raemdonck D, Erasmus M, Dark J, Love R, et al. International Society for Heart and Lung Transplantation donation after circulatory death registry report. J Heart Lung Transplant 2015;34:1278-8278. https://doi.org/10.1016/j. healun.2015.08.015.

[8] Erasmus ME, van Raemdonck D, Akhtar MZ, Neyrinck A, de Antonio DG, Varela A et al. DCD lung donation: donor criteria, procedural criteria, pulmonary graft function validation, and preservation. Transpl Int 2016;29:790-9. https://doi.org/10. 1111/tri.12738

[9] DerSimonian R, Laird N. Meta-analysis in clinical trials. Control Clin Trials 1986;7: 177-887. https://doi.org/10.1016/0197-2456(86)90046-2.

[10] Borenstein M, Hedges LV, Higgins JP. Introduction to meta-analysis. John Wiley \& Sons, Ltd: Chichester, UK; 2009.

[11] Higgins JPT. Measuring inconsistency in meta-analyses. BMJ 2003;27:557-607. https://doi.org/10.1136/bmj.327.7414.557.

[12] Higgins JPT, Thompson SG. Quantifying heterogeneity in a meta-analysis. Stat Med 2002;21:1539-5839. https://doi.org/10.1002/sim.1186.

[13] Hozo SP, Djulbegovic B, Hozo I. Estimating the mean and variance from the median, range, and the size of a sample. BMC Med Res Methodol 2005:5-13. https://doi.org/ 10.1186/1471-2288-5-13.

[14] R Core Team. R: a language and environment for statistical computing. R Foundation for Statistical Computing; Vienna, Austria, 2015: Available online: http://www.Rproject.org/ 
[15] Puri V, Scavuzzo M, Guthrie T, Hachem R, Krupnick AS, Kreisel D, et al. Lung transplantation and donation after cardiac death: a single Center experience. Ann Thorac Surg 2009;88:1609-14. https://doi.org/10.1016/j.athoracsur.2009.06.039.

[16] De Oliveira NC, Osaki S, Maloney JD, Meyer KC, Kohmoto T, D'Alessandro AM, et al. Lung transplantation with donation after cardiac death donors: long-term followup in a single center. J Thorac Cardiovasc Surg 2010;139:1306-506. https://doi. org/10.1016/j.jtcvs.2010.02.004.

[17] Van De Wauwer C, Verschuuren EAM, van der Bij W, Nossent GD, Erasmus ME. The use of non-heart-beating lung donors category III can increase the donor pool. Eur Cardio-Thoracic Surg 2011;39:e175-805. https://doi.org/10.1016/j.ejcts.2011.01.035.

[18] Levvey BJ, Harkess M, Hopkins P, Chambers D, Merry C, Glanville AR, et al. Excellent clinical outcomes from a national donation-after-determination- of-cardiac-death lung transplant collaborative. Am J Transplant 2012;12:2406-13. https://doi.org/ 10.1111/j.1600-6143.2012.04193.x.

[19] Sabashnikov A, Patil NP, Popov AF, Soresi S, Zych B, Weymann A, et al Long-term results after lung transplantation using organs from circulatory death donors: a propensity score-matched analysis. Eur J Cardio Thoracic Surg 2016;49:46-53. https:// doi.org/10.1093/ejcts/ezv051.

[20] Ruttens D, Martens A, Ordies S, Verleden SE, Neyrinck AP, Vos R, et al. Short- and long-term outcomes after lung transplantation from circulatory-dead donors: a single-Center experience. Transplantation 2017;101:2691-4691. https://doi.org/10. 1097/TP.0000000000001678.

[21] Costa J, Shah L, Robbins H, Raza K, Sreekandth S, Arcasoy S, et al. Use of lung allografts from donation after cardiac death donors: a single-Center experience. Ann Thorac Surg 2018;105:271-871. https://doi.org/10.1016/j.athoracsur.2017.07.023.

[22] Inci I, Hillinger S, Schneiter D, Opitz I, Schuurmans M, Benden C, et al. Lung transplantation with controlled donation after circulatory death donors. Ann Thorac Cardiovasc Surg 2018;24:296-302. https://doi.org/10.5761/atcs.oa.18-00098.
[23] Barbero C, Messer S, Ali A, Jenkins DP, Dunning J, Tsui S, et al. Lung donation after circulatory determined death: a single-Centre experience. Eur J Cardio-Thoracic Surg 2019;55:309-15. https://doi.org/10.1093/ejcts/ezy254.

[24] Oto T. Lung transplantation from donation after cardiac death (non-heart-beating) donors. Gen Thorac Cardiovasc Surg 2008;56:533-833. https://doi.org/10.1007/ s11748-008-0315-y.

[25] Wigfield C. Donation after cardiac death for lung transplantation. Curr Opin Organ Transpl 2014:19:455-955. https://doi.org/10.1097/MOT.0000000000000115.

[26] Krutsinger D, Reed RM, Blevins A, Puri V, De Oliveira NC, Zych B, et al. Lung transplantation from donation after cardiocirculatory death: a systematic review and meta-analysis. J Heart Lung Transplant 2015;34:675-845. https://oi.org/10.1016/ j.healun.2014.11.009.

[27] Machuca TN, Mercier O, Collaud S, Tikkanen J, Krueger T, Yeung JC, et al. Lung transplantation with donation after circulatory determination of death donors and the impact of ex vivo lung perfusion. Am J Transplant 2015;15:993-1002. https://doi. org/10.1111/ajt.13124.

[28] Saltelli A. Sensitivity analysis for importance assessment. Risk Anal 2002;22: 579-909. https://doi.org/10.1111/0272-4332.00040.

[29] Takada M, Nadeau KC, Hancock WW, Mackenzie HS, Shaw GD, Waaga AM, et al. Effects of explosive brain death on cytokine activation of peripheral organs in the rat. Transplantation 1998;65:1533-4233. https://doi.org/10.1097/00007890199806270-00001.

[30] Binns OA, DeLima NF, Buchanan SA, Nichols GE, Cope JT, King RC, et al. Impaired bronchial healing after lung donation from non-heart-beating donors. J Heart Lung Transplant 1996;15:1084-9284.

[31] Costa J, Benvenuto LJ, Sonett JR. Long-term outcomes and management of lung transplant recipients. Best Pract Res Clin Anaesthesiol 2017;31:285-975. https:// doi.org/10.1016/j.bpa.2017.05.006 